\title{
Klimaforschung in der Schweiz
}

Zum 70. Geburtstag von Max Schüepp

\section{Vorwort}

Nach einer Blütezeit Ende des letzten und zu Beginn dieses Jahrhunderts trat die Klimatologie in das zweite Glied zurück. Ihre Schwester, die Meteorologie, entfaltete sich besonders nach den beiden letzten Weltkriegen, begünstigt durch instrumentelle und übermittlungstechnische Fortschritte.

Der wirtschaftliche Aufschwung der Nachkriegszeit rief nach neuen Entscheidungsunterlagen für die Raumplanung und den Umweltschutz. Die traditionelle Klimatologie konnte den an sie gestellten neuen Anforderungen nicht mehr voll genügen. Wichtig gewordene statistische GröBen fehlten, sowohl für einzelne Klimaelemente wie auch für Beziehungswerte mehrerer Elemente untereinander.

Dank zusätzlicher Erweiterungen auf dem Gebiet der Meßtechniken, elektronischer Datenverarbeitungsanlagen und verbesserter physikalischer Simulationsmodelle atmosphärischer Vorgänge stieg vor allem im letzten Jahrzehnt das Interesse an neuen klimatologischen forschungsergebnissen. Diese erfreuliche Entwicklung in der Klimatologie ist durch die vielseitigen Kenntnisse und Interessen unseres verehrten Kollegen und Lehrers, Max Schüepp, reich befruchtet worden.

Das vorliegende Heft öffnet einen Einblick in einige ausgewählte Arbeitsgebiete der Klimaund Atmosphärenforschung in der Schweiz. Im ersten Beitrag legt W. Kirchhofer das neue Kartenwerk «Klimaatlas der Schweiz» vor. Es ergänzt die seit 1959 unter der Leitung von
M. Schüepp an der Schweizerischen Meteorologischen Anstalt in Zürich erscheinenden Tabellenwerke und Abhandlungen zur neuen «Klimatologie der Schweiz».

Eine neuartige, fast unerschöpfliche Datenquelle haben die Satelliten erschlossen. M. Winiger, G. Nejedly und Z.Schwab unterrichten über deren Einsatzmöglichkeiten beim Erkennen regionaler Abweichungen vom bekannteren großräumigen Klima, welche durch die vielgestaltigen Geländeformen erzeugt werden.

Für den Raum Basel-Juranord- und Schwarzwaldsüdseite spielen für Fragen der noch verantwortbaren weiteren Belastbarkeit der Luftschichten über dieser Wohn- und Industrieregion auch bessere Kenntnisse seiner Lokalwindsysteme eine wichtige Rolle. Diesem überraschend komplexen Aspekt widmet sich der Beitrag von W. Schüepp.

Auf noch kleinere Luftvolumina beschränken sich ungeschickterweise recht häufig die anthropogen bedingten schädlichen Beeinflussungen über größeren Siedlungen. Hier stellen die von H. Wanner, P. Berlincourt und R. Rickli erarbeiteten interdisziplinären Erkenntnisse wesentliche Entscheidungshilfen dar, die heute auch auf politischer Ebene nicht mehr vernachlässigt werden dürfen.
Gian Gensler

Schweizerische

Meteorologische Anstalt Postfach, 8044 Zürich 